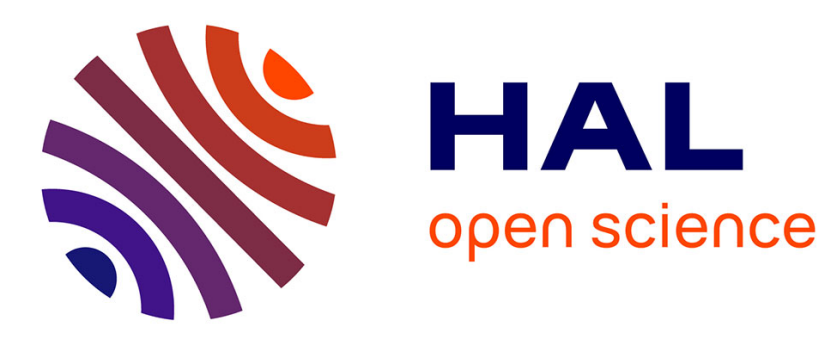

\title{
Percolation of superconducting "walls" in the ferromagnet HoMo 6S8
}

J.L. Genicon, J. P. Modon Danon, R. Tournier, O. Peña, R. Horyn, M. Sergent

\section{To cite this version:}

J.L. Genicon, J. P. Modon Danon, R. Tournier, O. Peña, R. Horyn, et al.. Percolation of superconducting "walls" in the ferromagnet HoMo 6S8. Journal de Physique Lettres, 1984, 45 (24), pp.1175-1181. 10.1051/jphyslet:0198400450240117500 . jpa-00232466

\section{HAL Id: jpa-00232466 https://hal.science/jpa-00232466}

Submitted on 1 Jan 1984

HAL is a multi-disciplinary open access archive for the deposit and dissemination of scientific research documents, whether they are published or not. The documents may come from teaching and research institutions in France or abroad, or from public or private research centers.
L'archive ouverte pluridisciplinaire HAL, est destinée au dépôt et à la diffusion de documents scientifiques de niveau recherche, publiés ou non, émanant des établissements d'enseignement et de recherche français ou étrangers, des laboratoires publics ou privés. 
Classification

Physics Abstracts

$74.70 \mathrm{~L}-74.10-75.60 \mathrm{E}$

\title{
Percolation of superconducting " walls " in the ferromagnet $\mathrm{HoMo}_{6} \mathbf{S}_{8}$
}

\author{
J. L. Genicon, J. P. Modon Danon, R. Tournier \\ Centre de Recherches sur les Très Basses Températures (*), BP 166 X, 38042 Grenoble Cedex, France \\ O. Peña, R. Horyn $\left(^{+}\right)$and M. Sergent
}

Laboratoire de Chimie M:nérale B $\left({ }^{* *}\right)$, Université de Rennes I, 35042 Rennes Cedex, France

(Reçu le 3 août 1984, accepté le 24 octobre 1984)

\begin{abstract}
Résumé. - Nous avons observé, loin en dessous de la température de Curie ferromagnétique et après cyclage en champ magnétique, un état de résistance nulle dans un échantillon de $\mathrm{HoMo}_{6} \mathrm{~S}_{8}$ constitué de quelques monocristaux. Ce nouvel état supraconducteur est attribué à la percolation de " parois » supraconductrices.
\end{abstract}

Abstract. - A zero-resistance state has been observed, far below the ferromagnetic Curie temperature and after cycling in a magnetic field, in a bulk sample of $\mathrm{HoMo}_{6} \mathrm{~S}_{8}$ composed of a few single crystals. This new superconducting state is attributed to the percolation of superconducting " walls ".

\section{Introduction.}

Among the so-called " Chevrel phases ", $\mathrm{HoMo}_{6} \mathrm{~S}_{8}$ has been extensively studied in recent years [1]. We know that $\mathrm{HoMo}_{6} \mathrm{~S}_{8}$ becomes superconducting at $T_{\mathrm{c}_{1}}\left(T_{\mathrm{c}_{1}} \cong 1.5 \mathrm{~K}\right)$ but at a lower temperature $T_{\mathrm{c}_{2}}\left(T_{\mathrm{c}_{2}} \cong 0.6 \mathrm{~K}\right)$, it re-enters the normal state at the onset of long range ferromagnetic order. In a narrow temperature range $T_{\mathrm{c}_{2}}<T<T_{\mathrm{m}}\left(T_{\mathrm{m}} \cong 0.67 \mathrm{~K}\right)$, the superconductivity coexists with a modulated magnetic structure [2].

In these compounds, as in $\mathrm{ErRh}_{4} \mathrm{~B}_{4}$, the resistivity is much smaller in the ferromagnetic state than in the paramagnetic state. The possible occurrence of superconductivity inside "walls " has been investigated, from a theoretical point of view, to explain that difference.

The calculations of Tachiki et al. [3] show that, in the ferromagnetic state of re-entrant superconductors, the region inside the Bloch walls could become superconducting. Kasperczyk et al. [4], for their part, have studied a ferromagnetic superconductor having both a magnetic domain structure of the Kittel-type and a laminar structure of the normal regions.

(*) CNRS. Laboratoire associé à l'Université Scientifique et Médicale de Grenoble.

(**) Laboratoire associé au CNRS, $\mathrm{n}^{\circ} 254$.

$\left({ }^{+}\right)$Permanent address : Institute for Low Temperatures and Structure Research, Polish Academy of Sciences, 50-950 pl. Katedralny 1, 50-950 Wroclaw, Poland. 
Recently, a systematic study [5] has shown that the existence of superconducting " walls " in the ferromagnetic state of re-entrant superconductors was indeed possible. The samples that were studied were made of sintered powder. It was suggested that a zero resistance state could be observed in single crystals.

In this paper we present the first results obtained on a bulk sample composed of a few single crystals, showing the superconductivity of « walls ».

\section{Experimental results.}

2.1 SAMPle AND EXPERIMENTS. - Lately, Peña et al. [6] have succeeded in synthesizing single crystals of $\mathrm{HoMo}_{6} \mathrm{~S}_{8}$. For more details about the purity of the samples, refer to [6]. We have studied a polycrystalline sample made of a few single crystals. This sample was $2.5 \mathrm{~mm}$ long, $1 \mathrm{~mm}$ wide and weighted $7 \mathrm{mg}$. The sample was placed in the mixing chamber of a dilution refrigerator. Resistivity measurements were performed using a standard four-probe a.c. technique. Usually the current in the sample was $100 \mu \mathrm{A}$. A current of $1 \mathrm{~mA}$ has also been used which corresponds to a minimum detectable resistance of $0.01 \mu \Omega$. The magnetic field was applied perpendicular to the length of the sample and to the current of measurement. Due to technical problems, magnetization measurements were not performed in that experiment ; they are now in progress.

2.2 CoOLING IN WEAK FIELDS. - The resistance of our sample at $4.2 \mathrm{~K}$ is $1040 \mu \Omega$, corresponding to a resistivity of $50 \pm 20 \mu \Omega . \mathrm{cm}$, the error being due to the determination of the geometrical factor. The residual resistivity ratio $\left(R_{\mathrm{amb}} / R_{4.2}\right)$ is equal to 10.5 . In a separate run, we measured the resistance at $4.2 \mathrm{~K}$ and did not observe any detectable magnetoresistance at this temperature for applied magnetic fields up to $60 \mathrm{kOe}$. This absence of magnetoresistance shows that the Ho spins have no influence on the resistivity when they are progressively aligned in the field.

The sample was first cooled slowly (i.e. $\sim 10$ hours) in zero field and the measurements done on increasing the temperature. The results are shown in figure 1 (closed circles). The resistance is $30 \%$ lower in the ferromagnetic state than in the paramagnetic state. The critical temperature $T_{\mathrm{c}_{2}}$ is equal to $0.5 \mathrm{~K}$ and the superconducting transition temperature $T_{\mathrm{c}_{1}}=1.5 \mathrm{~K}$. These results are very similar to the ones obtained on sintered samples. The lower value of the resistance in the ferromagnetic state cannot be attributed to a spin contribution, which would be negligible as demonstrated by the absence of magnetoresistance at $4.2 \mathrm{~K}$. We think rather that, as in sintered samples, superconducting paths short-circuit some parts of the sample [5].

The same kind of measurements was done after cooling the sample in the remanent field of the superconducting coil (field of the order of 50 to $100 \mathrm{Oe}$ ). The results are shown in figure 1 (open

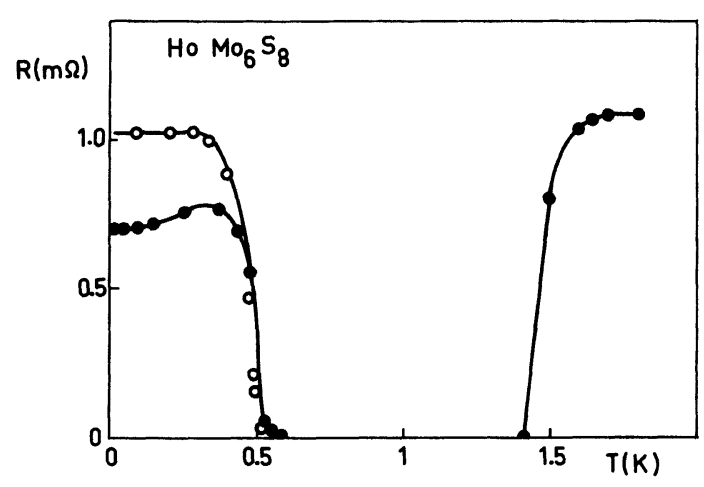

Fig. 1. - Variation of the resistance $R$ of $\mathrm{HoMo}_{6} \mathrm{~S}_{8}$ with temperature; (๑) sample cooled in zero field; (O) sample cooled in a small field of 50 to $100 \mathrm{Oe}$. 
circles). Below $0.4 \mathrm{~K}$, the resistance is constant and its value is nearly equal to the value measured in the paramagnetic state. This could signify that, by cooling under field, a large remanent magnetization has been achieved and that the internal induction, due to this large remanent magnetization has destroyed the superconductivity of the " walls ". An alternate explanation would be a preferential orientation of the "walls " around the direction of the external field and therefore perpendicular to the current. In this situation, the reduction of the resistivity due to superconducting paths could be negligible.

2.3 Zero Resistance STATE Below the Curie temperature. - Starting from the previous state (cooled down in a small field) and at $T=20 \mathrm{mK}$, the resistance remains approximately constant when a cycle $\left({ }^{1}\right)$ to a field of $20 \mathrm{kOe}$ is described. However, as the temperature is increased, the resistance becomes zero between $T=80 \mathrm{mK}$ and $T \cong 150 \mathrm{mK}$ (triangles, Fig. 2). Above this temperature, the sample goes back to the normal state and the resistance reaches its normal state value. Because the temperature was continuously increasing, the value of $150 \mathrm{mK}$ is only an approximate value (see Sect. 2.4). To recover the zero resistance state, we fixed the temperature at $130 \mathrm{mK}$ and described a cycle to a field of $20 \mathrm{kOe}$. In zero field, the resistance becomes zero again. The temperature was then lowered to $20 \mathrm{mK}$, the resistance still being zero. This zero resistance state is very stable at $20 \mathrm{mK}$ remaining constant for up to 3 days.

It is the first time, to our knowledge, that a zero resistance is observed in the ferromagnetic state of $\mathrm{HoMo}_{6} \mathrm{~S}_{\mathrm{R}}$. The way to get that zero resistance state is a clear indication of the existence of superconducting «walls ». By moving the walls under the effect of a magnetic field, they form a continuous superconducting path throughout the sample.

2.4 INFLUENCE OF TEMPERATURE AND TIME. - We have attempted to determine the temperature range where this new state exists. Starting from a zero resistance state at $T=20 \mathrm{mK}$, the temperature was gradually increased (squares, Fig. 2). For temperatures below $180 \mathrm{mK}$, this state is stable (at least at the scale of a few hours). For temperatures above $180 \mathrm{mK}$, we observe a destruction of the superconductivity as a function of time at a fixed temperature (shown by arrows, Fig. 2). This combined effect of time and temperature shows that the mobility of the "walls" increases rapidly for temperatures above $180 \mathrm{mK}$. For still higher temperatures $(T \cong 300 \mathrm{mK})$, it becomes difficult to distinguish between the effect of time and of temperature.

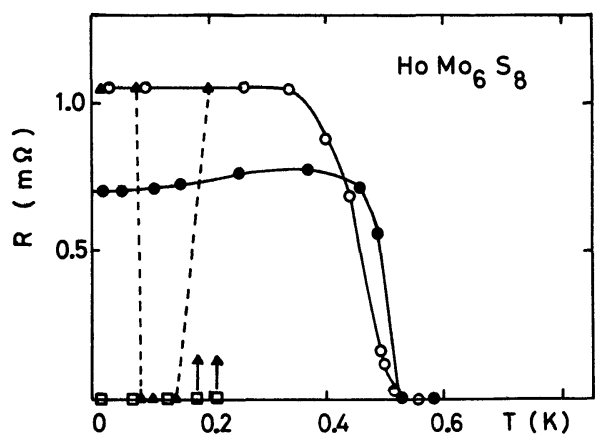

Fig. 2. - Resistance of $\mathrm{HoMo}_{6} \mathrm{~S}_{8}$ plotted versus temperature. (๑) and (O) : same as in figure 1 ; (४) after describing a cycle at $20 \mathrm{mK}$ and $20 \mathrm{kOe} ;(\square)$ after describing a cycle at $150 \mathrm{mK}$ and $20 \mathrm{kOe}$ then cooling down to $20 \mathrm{mK}$. The arrows mean a return to the normal state value, which is time dependent. All measurements were done on increasing the temperature.

( $\left.{ }^{1}\right)$ We define a cycle to a magnetic field as increasing the applied field from zero to a given value then decreasing it back to zero. 
The variation of $\Delta R=\left(R_{\mathrm{n}}-R\right) / R_{\mathrm{n}}$ is plotted versus time in figure 3, with a logarithmic scale for $\Delta R$. No simple variation is observed. Nevertheless a linear law can be approximated. The higher the temperature is, the faster is the recovery to the normal state.

2.5 INFLUENCE OF MAGNETIC FIELD. - Starting from a zero resistance state and at $T=25 \mathrm{mK}$, we have described a full cycle in magnetic field (Fig. 4). We first describe the curve 1 when increasing the field. The zero resistance state is destroyed in a field of $3.2 \mathrm{kOe}$ (defined as the field where $R=R_{\mathrm{n}} / 2$ ). The transition is rather broad and the resistance reaches its normal state value $R_{\mathrm{n}}$ in a field of $3.6 \mathrm{kOe}$. Then the resistance remains constant up to $20 \mathrm{kOe}$ and back to zero field. Then we reverse the direction of the field and we describe the curve 2 . An abrupt transition towards a zero resistance is observed at $1.6 \mathrm{kOe}$. The resistance does not reach a value equal to zero and goes back to $R_{\mathrm{n}}$, which is reached at $3.6 \mathrm{kOe}$. Curve 3 , symmetrical with curve 2 , is described when one reverses the field to the initial direction.

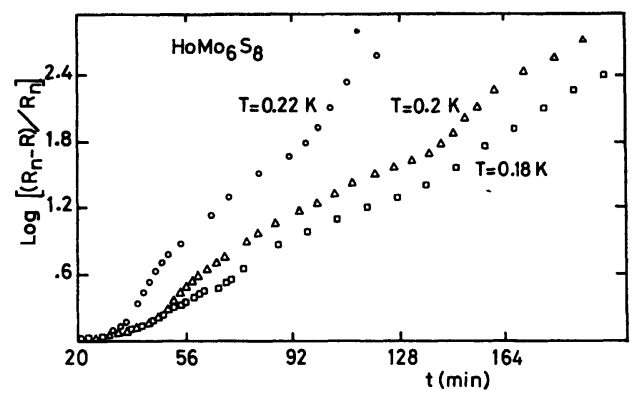

Fig. 3. - Variation of $\Delta R=\frac{R_{\mathrm{n}}-R}{R_{\mathrm{n}}}$ with time for different temperatures in $\mathrm{HoMo}_{6} \mathrm{~S}_{8}$.

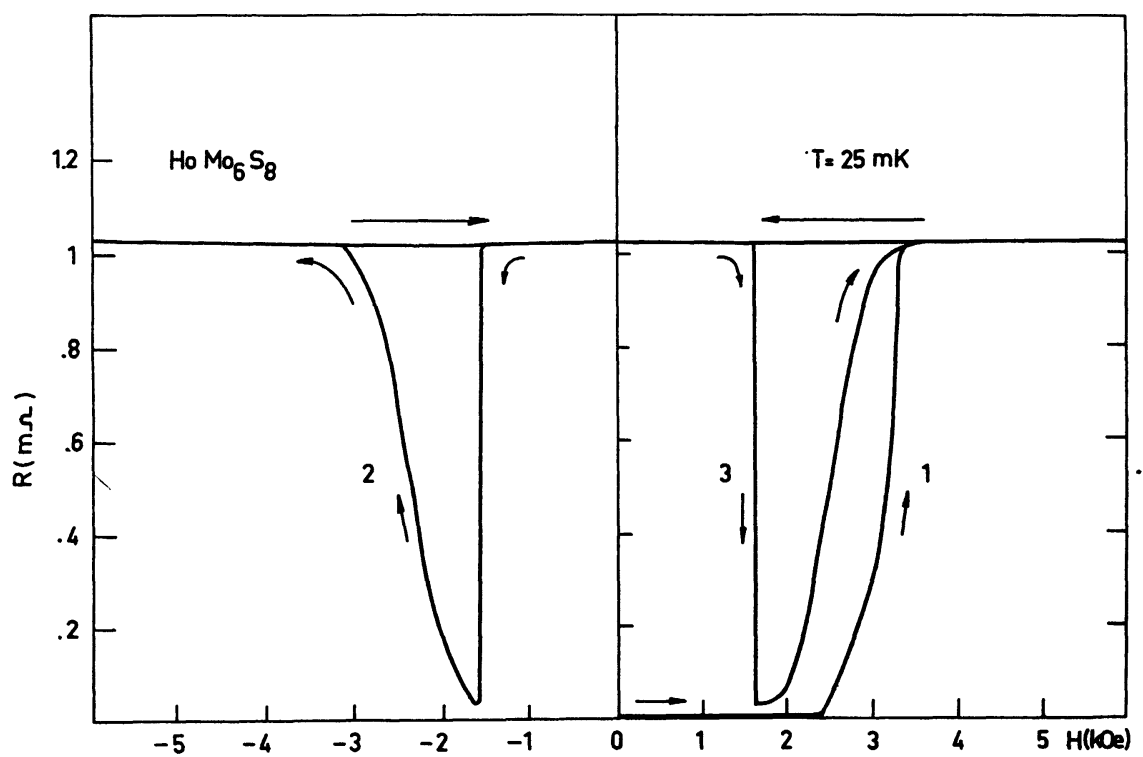

Fig. 4. - Variation of $R$ with magnetic field in $\mathrm{HoMo}_{6} \mathrm{~S}_{8}$. The different curves are traced in the order 1,2 and 3 as described in the text. 
Another way to get a zero resistance state is to start from $R_{\mathrm{n}}$ obtained after having described a cycle to $20 \mathrm{kOe}$ and to increase the field up to $1.6 \mathrm{kOe}$ in the opposite direction. The resistance reaches abruptly about $10 \%$ of $R_{\mathrm{n}}$ and becomes zero when one suppresses the field (Fig. 5).

As was shown in our previous work, the variation of resistance is related to the wall displacement by increasing the magnetization under a magnetic field. The zero resistance state must correspond to a state with a relatively small magnetization corresponding to many domains and " walls ". After having described a cycle to a $20 \mathrm{kOe}$ field (curve 2, Fig. 4), the remanent magnetization can be high without domains and "walls " leading to $R=R_{\mathrm{n}}$. The application of an inverse field lowers the internal induction and the magnetization by creating domains and " walls » leading to the zero resistance state.

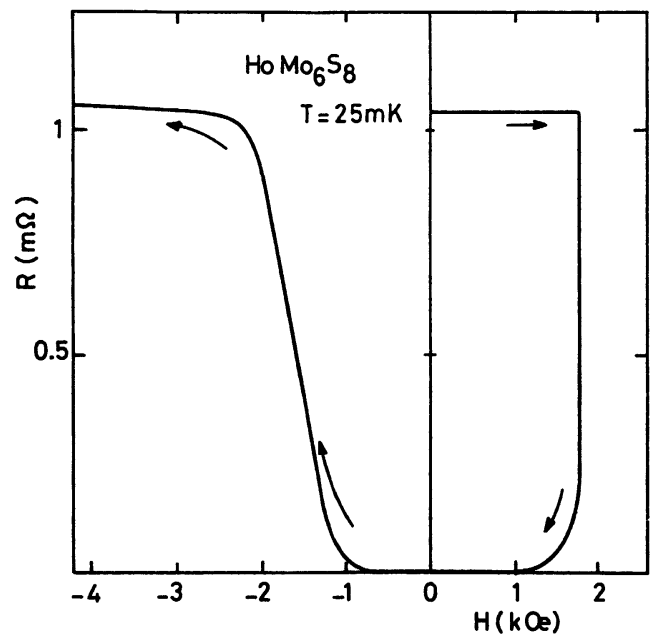

Fig. 5. - Variation of $R$ with magnetic field, starting from $R_{\mathrm{n}}$ and leading to a zero resistance. The curve on the left hand side is the same as curve 1 in figure 4.

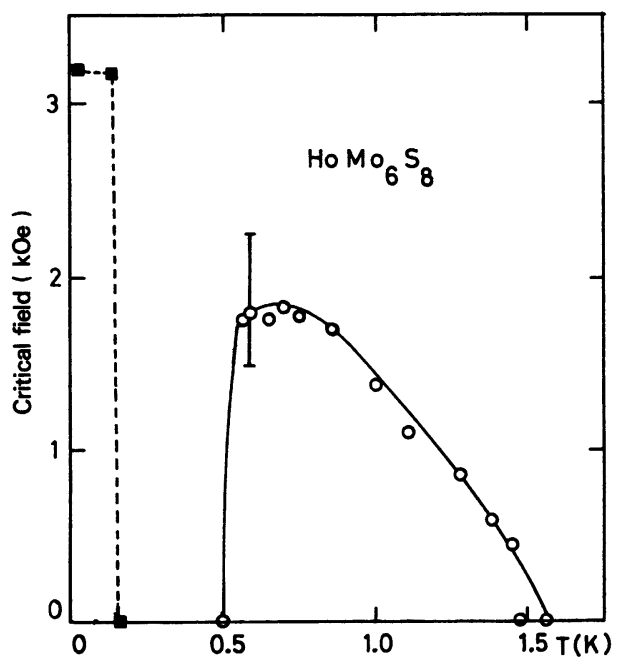

Fig. 6. - Critical field of $\mathrm{HoMo}_{6} \mathrm{~S}_{8}:(0)$ in the superconducting region, ( $(\boldsymbol{D})$ in the zero resistance state. The end points of the bar denote the field at which $R$ is $90 \%$ and $10 \%$ of $R_{\mathrm{n}}$. 
2.6 CRITICAL FIELD. - We have plotted in figure 6 the critical field measured in the superconducting regions and in the zero resistance state. The critical field has been defined as the field at which $R=R_{\mathrm{n}} / 2$.

The end points of the bar denote the field at which $R$ is $90 \%$ or $10 \%$ of $R_{\mathrm{n}}$. In the superconducting region, the value of the critical field is nearly the same as the one measured on sintered samples [5]. However, the critical field for the zero resistance state is much larger. From the slope $\left(\mathrm{d} H_{\mathrm{c}_{2}} / \mathrm{d} T\right)$ for $T=T_{\mathrm{c}}$, we can deduce a value for $H_{\mathrm{c}_{2}}(0)$ equal to $3.5 \mathrm{kOe}$, which would be obtained for the orbital limit without ferromagnetism. The critical field of the zero resistance state at $20 \mathrm{mK}(3.2 \mathrm{kOe})$ is nearly equal to this value.

\section{Comments and conclusions.}

We have succeeded in creating a zero resistance state below $180 \mathrm{mK}$ in $\mathrm{HoMo}_{6} \mathrm{~S}_{8}$. At $20 \mathrm{mK}$, one can observe either a normal state or a zero resistance state according to the history for field and temperature. The critical field is much higher in the zero resistance state than in the coexistence region between 0.5 and $0.6 \mathrm{~K}$. At $20 \mathrm{mK}$, the critical field is nearly equal to the value that one can deduce from the slope $\left(\mathrm{d}_{\mathrm{c}_{2}} / \mathrm{d} T\right)$ in the paramagnetic state.

At low temperature, in that new state, the superconductivity behaves as if the rare earths were paramagnetic and seems to ignore the existence of a magnetization either on a macroscopic scale (ferromagnetic phase) or on a microscopic scale (modulated phase). Such a phenomenon is only conceivable if the superconductivity takes place in " walls ".

Such an hypothesis is confirmed by the observation, at low temperature, of a ferromagnetic state with a value of the resistance lower than in the paramagnetic state. The fact that by cycling to a field lower than the critical field leads to a zero resistance state shows that the percolation can only take place after moving the "walls " in a magnetic field.

The value of the critical field at $20 \mathrm{mK}$ is lower in the case of single crystals than in the case of sintered powders. What we define as the critical field does not necessarily coincide with the true critical field for the superconductivity of the " walls ". It may only be the field at which the continuous superconducting path is broken due to the displacement of the « walls ".

Recent susceptibility measurements by Holtzberg et al. [7] on $\mathrm{Ho}_{2 / 3} \mathrm{Mo}_{6} \mathrm{~S}_{8}$ single crystals show that this compound is highly anisotropic. Neutron diffraction measurements have been performed by Quezel et al. [8] on a single crystal coming from the same batch as our sample. They show that, at low temperature, the magnetization points in the [111] direction with a structure of ferromagnetic microdomains of 1000 to $1500 \AA$.

In the presence of such a high anisotropy, the Bloch walls may be very narrow. The occurrence of superconductivity over a few interatomic distances seems difficult to imagine. For our part, we think that the superconducting "walls " are thicker, but move as the ferromagnetic walls. Such a superconducting-ferromagnetic structure is conceivable if the critical field is higher than the ferromagnetic induction, as observed in sintered samples.

More thorough studies, as a function of crystal orientations, are necessary to obtain a better understanding of this zero resistance state in a ferromagnet.

\section{References}

[1] For a general review, see Superconductivity in Ternary Compounds, Vol. II, Maple and Fischer Eds (Springer-Verlag, Berlin, Heidelberg, New-York) 1982.

[2] Lynn, J. W., Shirane, G., Thomlinson, W., Shelton, R. N., Moncton, D. E., Phys. Rev. B 24 (1981) 3817.

[3] Tachiki, M., Kotani, A., Matsumoto, H., Umezawa, H., Solid State Commun. 32 (1979) 599.

[4] KasperczyK, J., Koslowski, G., Tekiel, P., Solid State Commun. 44 (1982) 663. 
[5] Genicon, J. L., Laaboudi, B., Tournier, R., Chevrel, R., Sergent, M., J. Physique 45 (1984) 1079.

[6] Peña, O., Horyn, R., Potel, M., Padiou, J., Sergent, M., to appear in J. Less Common Metals (1984); Horyn, R., Peña, O., Sergent, M., to appear in J. Less Common Metals.

[7] Holtzberg, F., Laplaca, S. J., McGuire, T. R., Webb, R. A., J. Appl. Phys. 55 (1984) 2013.

[8] Quezel, S., Burlet, P., Roudaut, E., Rossat-Mignot, J., Benoit, A., Flouquet, J., Peña, O., Horyn, R., Chevrel, R., Sergent, M., Proceedings of « Journées Matériaux Supraconducteurs », to be published in Annales de Chimie, Sciences des Matériaux. 